\title{
Dietary Flavonoids and Colorectal Cancer: Evidence from Epide- miological Studies
}

\author{
Katrin Sak* \\ NGO Praeventio, Tartu, Estonia
}

*Corresponding author: Katrin Sak, NGO Praeventio, Näituse 22-3, Tartu 50407, Estonia, Tel: +372-53-341-381, E-mail: katrin. sak.001@mail.ee

\begin{abstract}
Identification of dietary agents related to colorectal carcinogenesis is of high importance to decrease the global burden of this severe disorder. As flavonoids abundantly present in plant-based food items are suggested to exert various anticancer activities in numerous experimental models, evidence from epidemiological studies about association between dietary intake of these plant secondary metabolites and the risk of colorectal cancer was compiled and is discussed in this review article. Several case-control studies have demonstrated that dietary intake of selected subclasses of flavonoids and also some individual compounds can be inversely associated with colorectal cancer risk. However, these findings are not in complete agreement and are mostly not confirmed also by the results of prospective cohort studies. Several reasons are proposed to explain discrepancies and inconsistencies of findings obtained from different works, starting from the dependence of flavonoid content on cultivation conditions of plants and methods of food processing to the factors related to study design. At that, food sources of flavonoids (tea or non-tea flavonoids) can affect the role of these polyphenolic agents on development of colorectal cancer and the potential protective effects may also depend on cancer subsites, i.e. tumors in proximal vs. distal colon or rectum. Therefore, associations between flavonoids intake and colorectal malignancies are complex and biological mechanisms and physiological importance of various established and still unknown factors certainly need more investigation. Also, further large-scale prospective cohort studies in different populations are required before any public health recommendations can be formulated for general population or at-risk individuals.
\end{abstract}

\section{Keywords}

Colorectal cancer, Colon cancer, Rectum cancer, Epidemiology, Dietary polyphenols, Flavonoids

\section{Introduction}

Colorectal cancer is one of the most frequently occurring cancers in the world, being the third most common neoplasms in men and the second one among women [1-3]. Colorectal cancer is a fourth leading cause of cancer-related death worldwide affecting somewhat more men than women [2,4]. Despite some decrease in colorectal cancer rate in United States during the last 15 years, the incidence in other countries has remained stable or has been gradually increased [2]. The decline observed in United States can be attributed to the improvements in screening programs and removal of premalignant adenomas [2]. The most of colorectal malignancies occur in non-hereditary forms with the major risk factors including low level physical activity, unhealthy dietary choices, obesity, smoking and also lack or paucity of national screening programs $[2,5]$. Therefore, prevention is a key strategy to decrease the colorectal cancer incidence, whereas diet as a modifiable factor in human's behavioral pattern might be a crucial determinant [5-7].

Although it has been proposed that dietary choices can be responsible even for $70-90 \%$ of all colorectal cancer cases [8], identifying specific nutrients accounting for the decrease in cancer risk has proven to be rather difficult [9]. Numerous studies have suggested that high consumption of plant-derived food items, such as various fruits and vegetables can be implicated in the lower susceptibility to colorectal carcinogenesis [6,9-12]. At that, certain phytochemicals in these plant-based products, i.e. flavonoids have attracted an enormous interest in the recent decades $[6,10]$. Flavonoids, abundant in

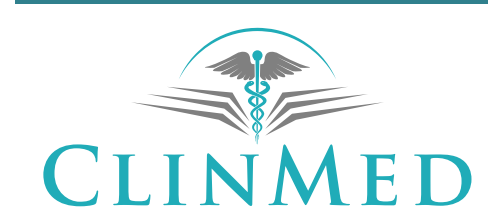

INTERNATIONAL LIBRARY 
vegetables, fruits, spices, herbs, seeds, grains, tea and wine, exert different biological functions and may play a substantial role in the protection against colorectal cancer [7,11-13]. These plant secondary metabolites reveal antioxidant, anti-inflammatory, antimicrobial, antiproliferative, proapoptotic, antiangiogenic, and antimetastatic properties, modulating thus the initiation and progression of colorectal tumors $[6,12,14]$. The high structural diversity of flavonoids enables these polyphenolic compounds also to exhibit diverse health benefits $[10,12,15]$. For example, evidence has been reported about the reduction of colorectal cancer risk in animals with high intake of soy isoflavones $[3,16]$. However, the exact biochemical mechanisms behind these effects are still not fully understood. Moreover, extrapolation of the results obtained from in vitro basic research or in vivo animal studies to the colorectal cancer etiology in humans requires caution as the concentrations of flavonoids used in experimental works have been considerably higher than those achieved via the habitual dietary consumption $[6,9,15,17]$. Secondly, the bioavailability of flavonoids is generally poor allowing plasma concentrations of these substances usually not more than some micromolar, which remain about tenfold lower than the doses found to be active in experimental studies with colorectal cancer cell lines $[17,18]$. Third, as a result of bioconversion occurring in the small intestine and liver, various metabolites of flavonoids with potentially altered biological activities enter into the bloodstream and reach target sites $[9,19]$. Nevertheless, of all the human tissues, the intestine can be exposed to higher doses of flavonoids than any other tissues in the body, expressing also their biological effects, including anticarcinogenic activities in loco $[10,12,17,18]$. Therefore, it is clear that the potential protective action of flavonoids against colorectal tumorigenesis certainly needs further proofs from epidemiological studies and from prospective interventional randomized trials $[5,6]$. Current epidemiological findings about the associations between the dietary consumption of flavonoids and the risk of colorectal cancer have still been limited, mixed and inconsistent [3,5,7,10-12,15,17,18,20].

In this small review article, epidemiological data published to date about the relationships between intake of flavonoids, their subclasses and individual compounds with the susceptibility to colorectal carcinogenesis were compiled, both from case-control and prospective cohort studies. These findings are presented, considering the subsites of tumors (colon vs. rectum, proximal colon vs. distal colon) as well as potential confounding factors, such as age, gender, obesity or smoking status of participants. Studies measuring the different biomarkers in body fluids are also included in the paper. All these data are presented in the Table 1, Table 2 and Table 3 and are further discussed in the following subsections. In addition, the possible reasons of inconsistencies in the results of different epidemiological studies are also treated.

\section{Associations between Flavonoid Intake and Col- orectal Cancer Risk}

\section{Case-control studies}

Several case-control studies with different results have been reported (Table 1 ). No significant interaction was found between intake of total flavonoids and colorectal cancer risk among Italian [21] and Chinese men and women [11] for both colon and rectal tumors. On the contrary, consumption of high doses of total flavonoids was inversely associated with colorectal neoplasms in Spanish population with $41 \%$ decrease in cancer risk being substantially similar for the tumors in colon and rectum ( $45 \%$ and $36 \%$ reduction, respectively, for the highest versus the lowest quartiles) [18]. This discrepancy can be somewhat explained by the fact that differently from the Spanish study, the Italian work did not include proanthocyanidins in total flavonoids $[18,21]$.

According to their chemical structure, dietary sources and biological activity, flavonoids can be subdivided into six major subclasses: Flavones, flavonols, flavanones, catechins or flavanols, isoflavones, and anthocyanidins $[7,14]$. Intake of flavones was shown to be inversely associated with the risk of colorectal cancer in Italian population with $22-23 \%$ reduction in the incidence rate of both colon and rectal neoplasms [21]. Significant inverse relationship was also found between flavone intake and colorectal cancer risk among Spanish men and women revealing $41 \%$ decrease in cancer risk being slightly stronger for colon (46\%) than rectal tumors (31\%) [18]. Similarly, the study performed with Chinese population demonstrated about $45 \%$ lowering in the colorectal cancer risk with high intake of dietary flavones, whereas the risk reduction was similar for coIon and rectal malignancies [11]. However, the results of the Study of Colorectal Cancer in Scotland (SOCCS) did not confirm the protective action of flavones against colorectal cancer, showing no association between flavone intake and the risk of colorectal tumorigenesis in women or men, smokers or non-smokers, colon or rectal cancer [20]. The reasons for this discrepance are unclear. It is possible that limited dietary sources of flavones (celery, herbs) can lead to a loss of study power [20]. However, other reasons can include the less complete nutritional database information on flavones resulting in greater misclassification and different biological activities of different individual flavones.

Increased dietary consumption of flavonols was related to about 35\% lowered colorectal cancer incidence (both colon and rectal malignancies) in Italian population [21]. Likewise, high intake of flavonols was associated with almost $30 \%$ reduction of colorectal cancer risk among Scottish men and women, being greater for non-smokers than smokers [20]. At that, the most common flavonoid in the diet, quercetin, abundant in onions and apples, was related to one-third decreased risk of both colon and rectal tumors [20]. However, another study conducted in Amer- 
ican population suggested a protective effect of quercetin only against carcinogenesis in proximal (and not distal) colon [22]. A more recent case-control study performed in Scottish population found no significant relationship between total dietary flavonols and colorectal cancer risk [12]. Although this study had different age distribution of participants and smaller sample size than the work of Theodoratou, et al. [20], statistically significant inverse association with colon, but not rectal tumors, still revealed if the analysis was confined to non-tea flavonols [12]. Also, whereas high consumption of quercetin from non-tea dietary sources was related to about twofold decrease in colon (not rectal) cancer risk, no relationships were detected for dietary intake of two other common food flavonols, kaempferol and myricetin [12]. Suggestive, but statistically non-significant, inverse association was detected also between flavonols and colon (not rectal) cancer risk in Spanish population [18]. In the study performed with Chinese subjects, a significant inverse association between dietary consumption of flavonols and developing of colorectal neoplasms was found only for men, and not women, with a $49 \%$ decrease in cancer incidence [11].

Most of the published case-control studies conducted with European populations reported no significant associations between intake of flavanones and colorectal carcinogenesis $[18,19,21]$. This was shown to be true also for two major dietary flavanones, hesperetin and naringenin [20]. However, a significant inverse relationship was found between intake of flavanones and the risk of colorectal cancer in Chinese population with up to $80 \%$ decrease in tumorigenesis, for both colon and rectal neoplasms [11]. On the contrary, a weak tendency towards increased susceptibility to developing of colorectal malignancy with higher intake of flavanones was detected in a Scottish study [12]. It can be that the food sources of flavanones are somewhat different in Asian and European countries affecting the potential action of these dietary compounds. However, we can not exclude also the possibility that some genetic differences could be involved in this discrepancy altering colorectal cancer predisposition in Asian and European populations.

No significant relationships were observed between the risk of colorectal cancer and consumption of flavanols in Italian [21], Spanish [18], Scottish [12] or Chinese population [11]. Although findings from another Scottish study for total flavanols are consistent with null association, two individual flavanols, catechin and epicatechin, revealed a significant and dose-dependent inverse effect on the susceptibility to colorectal cancer with about $30 \%$ risk reduction of both colon and rectal tumors [20]. This decrease in risk was greater for non-smokers than smokers and the protective effect of catechin seemed to be slightly stronger among men than women [20].

Epidemiological findings about the role of isoflavones intake on the risk of colorectal cancer are rather inconsistent. No risk reduction was reported for English
[23] or Spanish subjects [18]. However, inverse association between increased dietary intake of isoflavones and developing of colorectal tumors was observed in studies conducted with Canadian [24], Italian [21] or Korean populations [25]. Among Korean women, the protective action of isoflavones was somewhat stronger among postmenopausal than premenopausal counterparts [25]. In stratification by tumor subsites, a significantly lowered risk for the highest consumption groups remained persistent for distal colon cancers in Korean men and rectal neoplasms in Korean women [25]. The same trends were also true for three individual isoflavones, daidzein, genistein and glycitein [25]. In a Japanese study, significant inverse relationship with dietary intake of isoflavones was demonstrated also for colorectal adenomas which are considered as precursor lesions of colorectal tumors [4]. This association was stronger among women than men and did not depend on the size, number or site of adenomas [4]. Differences in effects of isoflavones on colorectal carcinogenesis described in separate studies can mostly be attributed to the variability in amounts of soy food, and consequently also isoflavones, consumed. For example, daily intake of isoflavones in Japanese case-control study with colorectal adenomas was $39.75 \mathrm{mg}$, being only $0.21 \mathrm{mg}$ in Spanish colorectal cancer association study [18].

A decrease in colorectal (both colon and rectal) cancer risk was reported also for higher intake of anthocyanidins in an Italian [21] and Chinese case-control study [11], but not in a work conducted with Spanish men and women [18].

\section{Prospective cohort studies}

Despite promising epidemiological findings from several case-control works, results from prospective cohort studies are still much less encouraging (Table 2). No significant associations between intake of total flavonoids and colorectal cancer risk have been found in American [15,26], Finnish [27-29] or Dutch population [30]. Also, dietary consumption of flavones, flavonols, flavanones, flavanols and anthocyanidins was not related to developing of colorectal cancer (neither colon or rectal tumors) among American men recruited to the Health Professional Follow-up Study, American women in the Nurses` Health Study cohort [17] or Finnish men in the Kuopio Ischaemic Heart Disease Risk Factor Study [29]; thus, not supporting the hypothesis of a protective role of flavonoids against colorectal neoplasms. No appreciable associations were observed also for individual flavonols, such as kaempferol, myricetin and quercetin $[5,15,28,30]$. However, stratifying the analysis by Body Mass Index (BMI) revealed a significant inverse association between intake of kaempferol and myricetin and colorectal cancer risk among normal weight Dutch women with $\mathrm{BMI}<25$, in particular for colon tumors [5]. Likewise, although intake of total flavanols (or individual flavanols) was not related to the risk of colorectal 
carcinogenesis in the Netherlands Cohort Study, analyses stratified by BMI revealed significant inverse relationships for intake of total flavanols, catechin and epicatechin with rectal neoplasms among overweight men with $\mathrm{BMI} \geq 25$ [5]. Inverse associations were observed also for higher dietary consumption of total flavanols, catechin, epicatechin, epicatechin gallate, epigallocatechin, epigallocatechin gallate and gallocatechin with the risk of colorectal cancer, especially colon tumor, among normal weight women with $\mathrm{BMI}<25$ [5]. The authors of this Netherlands Cohort Study suggested that the influences of BMI on the role of flavonoids in colorectal carcinogenesis can be related to the action of Insulin-like Growth Factor 1 (IGF-1) that is secreted by adipose tissue and promotes malignant cell growth, whereas its level can be lowered by some flavanols, such as epigallocatechin gallate. As overweight postmenopausal women have higher estrogen levels as compared to their normal weight counterparts, effects of flavonoids in this subgroup might be overshadowed by potentially protective action of estrogens against colon carcinogenesis [5]. Increased intake of total flavanols was found to be significantly inversely associated with rectal (not proximal or distal colon) cancer risk among postmenopausal American women in the lowa Womens Health Study [31]. However, these findings are somewhat different from the results of case-control studies described in the previous subsection.

Observational association studies with isoflavones have been performed to date only with Asian populations (Chinese or Japanese) [3,16,32], probably due to the much higher intake of isoflavone-rich soy foods in these countries. While a suggestive inverse relationship was observed between higher intake of isoflavones and colorectal cancer risk in primarily postmenopausal Chinese women in the Shanghai Women's Health Study [16], no significant association was found between isoflavone intake and colorectal cancer risk in either Japanese men or women in the Japan Public Health Center-based prospective study [3]. However, the malignancy risk in proximal colon (not distal colon or rectum) was significantly reduced with increasing intake of isoflavones among Japanese men (and not women), revealing a $45 \%$ decrease [3]. At that, the menopausal status of women did not change the trends of association [3]. Again, these findings from prospective cohort studies are not in good accordance with the results obtained from case-control works, showing that further large-scale observational studies in different populations are highly needed to delineate the role of certain flavonoid subclasses and individual flavonoids on developing of malignant disorders in different anatomical subsites of colorectum.

\section{Biomarker studies}

In addition to retrospective or prospective assessment of dietary intake of different flavonoids, the search and estimation of biomarkers has become attractive, especially through the evaluation of the bioavailability and fate of flavonoids in the human body. It is well known that flavonoids undergo an extensive bioconversion in the small intestine and liver, as result of which different conjugates with potentially altered biological activities enter the bloodstream and reach target tissues [19]. However, only two studies have been performed to date on association between the levels of flavonoids in the body fluids and colorectal cancer risk (Table 3). The first of these studies was conducted with Chinese men within the Shanghai Cohort Study by investigating the urinary flavanol derivatives [33]. In this work, statistically significant inverse association with colon (but not rectal) cancer risk was found for subjects with high urinary doses of epigallocatechin and 4`-O-methyl epigallocatechin, revealing about $60 \%$ decrease in colon cancer incidence and supporting thus the hypothesis of protective effects of flavanols as chemopreventive agents against colon tumorigenesis [33]. The second study investigating relationships between biomarkers of flavonoids and colorectal cancer risk was performed with English men and women within the European Prospective Investigation into Cancer and Nutrition Norfolk cohort by measuring the levels of different isoflavones in serum and urine [34]. As a consequence, no significant associations were detected for total or individual isoflavones with the risk of colorectal tumors showing that exposure to isoflavones could not be related to colorectal malignancies, at least in doses consumed in this population [34]. With no doubts, further studies are needed to clarify the potential prediagnostic value of flavonoid biomarkers for estimation of susceptibility to colorectal carcinogenesis.

\section{Explanation for inconsistencies}

The discrepancies of epidemiological findings about the role of flavonoids in colorectal carcinogenesis cannot be easily resolved due to the multifactorial nature of associations. Epidemiological studies of flavonoids have long been complicated by unavailability of reliable nutrient databases on the content of flavonoids in different food items $[10,21]$. In 2003, the United States Department of Agriculture (USDA) announced first a flavonoid database which can be connected to food frequency questionnaires, promoting scientific community to perform association studies between dietary intake of different flavonoid subclasses and susceptibility to carcinogenesis $[10,20]$. This database has been updated for several times and some inconsistencies in epidemiological findings can be explained by the use of older versions of food composition databases [18,22]. Also, questionnaires used in different studies are often not specifically designed for assessment of flavonoid consumption, lacking some particular foods rich in certain polyphenols, such as herbs or herbal remedies, and can therefore lead to incomplete estimation of flavonoid intake $[6,7,15,17,18,20,21]$. In addition, the content of flavonoids in plant-derived food items may vary depending on numerous factors, including species variety, 
climatic and agricultural conditions, seasonal variation, ripeness, storage and processing of food products; not included in databases $[6,10,15,17,21]$. These aspects question the adaptability of USDA database in the European or Asian diets [21]. Also, extrapolation of findings across populations requires caution.

Only a small number of flavonoids have been evaluated in epidemiological studies and different works considered somewhat different combinations of flavonoids and subclasses of flavonoids $[7,10,12,17]$. For example, whereas Zamora-Ros, et al. treated isoflavones as the sum of daidzein, genistein, glycitein, biochanin A and formononetin [18], Rossi, et al. considered isoflavones as the sum of genistein and daidzein [21]. Also, Zamora-Ros, et al. and Nimptsch, et al. counted in flavanones eriodictyol, hesperetin and naringenin $[17,18]$, whereas Rossi, et al. and Theodoratou, et al. did not include the intake of eriodictyol in total flavanones [20,21]. Differences appear also in counting of isorhamnetin in total flavonols, besides the sum of kaempferol, myricetin and quercetin $[17,18,20,21]$. The dietary intake of eriodictyol and isorhamnetin is probably low, but can still cause inconsistencies in epidemiological findings when exerting stronger biological activity. Discrepancies in epidemiological results can be produced also by variations in sources of certain flavonoids in separate studies. Growing evidence suggests that higher intake of flavonols, including quercetin, only from non-tea sources (i.e. from fruits and vegetables) may be related to decreased risk of colon carcinogenesis $[11,12,22]$, probably caused by some other substances in tea. As phytochemicals are present in different mixtures within the food matrix, various interactions with other food ingredients are possible, affecting also the role of flavonoids in carcinogenesis process.

Differences in epidemiological results between dietary consumption of flavonoids and colorectal cancer risk can be derived also by variability of habitual intake levels of flavonoids among different populations [11]. For example, it is well accepted that daily consumption of isoflavone-rich soy foods is considerably lower in Western countries than in Asian populations and this difference can be even more than hundredfold $[9,16,18,25]$. Therefore, it is possible that in some cases the null associations might be caused by insufficient intake range of certain flavonoids to reach the doses that would be protective against colorectal carcinogenesis $[7,10,17,20]$. The timing of exposure to flavonoids might be another crucial factor as early intake of certain flavonoids could probably influence the formation of adenomas, preventing thus the development of colorectal tumors [17].

Most flavonoids are present in food products in the form of glycosides which after hydrolysis to respective aglycones in the small intestine absorb through the intestinal membrane and undergo an extensive bio- conversion to various metabolites $[15,17,19]$. Microorganisms in the colon make an important contribution to this process and interindividual variations in colonic microflora may affect the amount and nature of conjugates and therefore modulate also the possible anticarcinogenic action $[10,15,17,20]$. As gastrointestinal tract is directly exposed to higher doses of flavonoids than other tissues in the human body, the beneficial effects of these plant-derived substances might be more effective at that site. The plasma concentrations of flavonoids rarely exceed some micromolar level after habitual dietary intake $[9,22]$. However, the bioavailability of flavonoids considerably depends on dietary sources, food preparation techniques, and also other components in food matrix $[10-13,22]$. For example, quercetin absorbs much more rapidly from onions than from tea, being intermediate from apples [11]. Assessment of food intake can not consider all these aspects, i.e. interindividual variations in bioavailability and metabolism of flavonoids; therefore, measurement of biomarkers in urine or serum might provide more relevant insight into the association between flavonoids intake and colorectal cancer risk [34]. However, these biomarkers reflect only very recent dietary consumption due to short elimination time of flavonoids from the body (within some hours) and could not provide a true picture about longterm dietary habits $[9,33]$. Thus, estimation of dietary intake using continuously updated flavonoid databases still remains a valuable measure for association studies.

Furthermore, inconsistencies in epidemiological findings can be caused also by the differences in study design. Case-control works may suffer from recall bias due to differential remembering of the dietary habits by colorectal cancer patients as compared to control subjects $[6,10,17,18,21]$. The other potential hazard of case-control studies is selection bias. Indeed, the dietary habits reported by hospital controls could not represent those characteristic for the general population $[11,21]$. Prospectively collected dietary data are probably free of differential errors caused by existence of tumor [10,1517,34]. However, the intake of flavonoids was often based on a single assessment at study baseline and this might not represent the dietary habits over several follow-up years [5]. Moreover, some studies included only a relatively small number of cancer cases possessing limited power to detect a relationship between intake of flavonoids and susceptibility to colorectal carcinogenesis $[10,18,20,21]$.

Although different risk factors for colorectal tumors were adjusted in most epidemiological studies, the possibility of uncontrolled or unknown residual confounding factors can not be excluded $[5,10,11,12,15,22]$. Higher intake of flavonoids is usually related to an overall healthy lifestyle that is in itself protective against development of colorectal neoplasm $[5,7,10]$. Moreover, bioactive agents in plant-based food items are highly correlated and complex pointing to the possibility that some other compo- 
nents or still unidentified compounds could also account for beneficial health effects $[6,10,12,22]$.

Last but not least, it is possible that the role of flavonoids in colorectal carcinogenesis can be affected also by interactions between diet and genes, including presene of single nucleotide polymorphisms. Such potential relationships certainly need further investigation and unraveling in the future.

\section{Conclusions and Further Perspectives}

Numerous experimental studies have demonstrated the anticancer activities of flavonoids in different colorectal cancer cell lines or animal models [14] encouraging to consider these plant secondary metabolites as promising chemopreventive agents. However, based on the epidemiological data published to date, no clear and consistent evidence can still be brought forth to support the hypothesis of protective action of increased intake of flavonoids against colorectal carcinogenesis. Although several case-control studies reported inverse associations between intake of certain flavonoid subclasses or individual compounds and colorectal cancer risk, prospective cohort studies did not generally find any significant relationships, with the exception of the lowa Women's Health Study that reported a $45 \%$ decrease in rectal cancer incidence with high intake of total flavanols among postmenopausal women [31] and the Japan Public Health Center-based prospective study that detected a $45 \%$ reduction in prevalence of proximal colon cancer with high intake of total isoflavones among men [3]. Although, body mass index can affect the role of some flavonols and flavanols on development of colorectal cancer, overweight or higher body fatness is in itself recognized as a risk factor for colorectal malignancies [5]. Nevertheless, based on several studies, flavonoids can be effective in reducing the risk of colorectal adenoma recurrence preventing thus also the subsequent tumorigenesis process $[7,35,36]$. It is clear that further large-scale studies in different populations with varying daily intake levels of flavonoids are highly needed before any clear trend of association can be brought forth and any public health recommendations created either for general public or at-risk subjects with increased predisposal to colorectal cancer. As diet is a potentially modifiable factor in human behavior identification of certain plant-based compounds to decrease colorectal cancer incidence may be an important strategy for primary prevention.

\section{Conflict of Interest}

None declare.

\section{References}

1. World Cancer Report (2014) Lyon: International Agency for Research on Cancer. In: Stewart BW, Wild CP, World Health Organization.

2. Favoriti P, Carbone G, Greco M, Pirozzi F, Pirozzi RE, et al. (2016) Worldwide burden of colorectal cancer: a review. Updates Surg 68: 7-11.
3. Akhter M, Inoue M, Kurahashi N, Iwasaki M, Sasazuki S, et al. (2008) Dietary soy and isoflavone intake and risk of colorectal cancer in the Japan public health center-based prospective study. Cancer Epidemiol Biomarkers Prev 17: 2128-2135.

4. Akhter M, Iwasaki M, Yamaji T, Sasazuki S, Tsugane S (2009) Dietary isoflavone and the risk of colorectal adenoma: a case-control study in Japan. Br J Cancer 100: 1812-1816.

5. Simons CC, Hughes LA, Arts IC, Goldbohm RA, van den Brandt PA, et al. (2009) Dietary flavonol, flavone and catechin intake and risk of colorectal cancer in the Netherlands Cohort Study. Int J Cancer 125: 2945-2952.

6. He X, Sun LM (2016) Dietary intake of flavonoid subclasses and risk of colorectal cancer: evidence from population studies. Oncotarget 7: 26617-26627.

7. Bobe G, Sansbury LB, Albert PS, Cross AJ, Kahle L, et al. (2008) Dietary flavonoids and colorectal adenoma recurrence in the Polyp Prevention Trial. Cancer Epidemiol Biomarkers Prev 17: 1344-1353.

8. Pericleous M, Mandair D, Caplin ME (2013) Diet and supplements and their impact on colorectal cancer. J Gastrointest Oncol 4: 409-423.

9. Messina M, Bennink M (1998) Soyfoods, isoflavones and risk of colonic cancer: $A$ review of the in vitro and in vivo data. Baillieres Clin Endocrinol Metab 12: 707-728.

10. Kocic B, Kitic D, Brankovic S (2013) Dietary flavonoid intake and colorectal cancer risk: evidence from human population studies. J BUON 18: 34-43.

11. Xu M, Chen YM, Huang J, Fang YJ, Huang WQ, et al. (2016) Flavonoid intake from vegetables and fruits is inversely associated with colorectal cancer risk: a case-control study in China. Br J Nutr 116: 1275-1287.

12. Kyle JA, Sharp L, Little J, Duthie GG, McNeill G (2010) Dietary flavonoid intake and colorectal cancer: a case-control study. Br J Nutr 103: 429-436.

13. Hoensch HP, Kirch W (2005) Potential role of flavonoids in the prevention of intestinal neoplasia: a review of their mode of action and their clinical perspectives. Int J Gastrointest Cancer 35: 187-195.

14. Sak K (2014) Cytotoxicity of dietary flavonoids on different human cancer types. Pharmacogn Rev 8: 122-146.

15. Lin J, Zhang SM, Wu K, Willett WC, Fuchs CS, et al. (2006) Flavonoid intake and colorectal cancer risk in men and women. Am J Epidemiol 164: 644-651.

16. Yang G, Shu XO, Li H, Chow WH, Cai H, et al. (2009) Prospective cohort study of soy food intake and colorectal cancer risk in women. Am J Clin Nutr 89: 577-583.

17. Nimptsch K, Zhang X, Cassidy A, Song M, O'Reilly ÉJ, et al. (2016) Habitual intake of flavonoid subclasses and risk of colorectal cancer in 2 large prospective cohorts. Am J Clin Nutr 103: 184-191.

18. Zamora-Ros R, Not C, Guino E, Lujan-Barroso L, Garcia RM, et al. (2013) Association between habitual dietary flavonoid and lignan intake and colorectal cancer in a Spanish case-control study (the Bellvitge Colorectal Cancer Study). Cancer Causes Control 24: 549-557.

19. Sak K, Everaus H (2016) Nanotechnological approach to improve the bioavailability of dietary flavonoids with chemopreventive and anticancer properties. In: Grumezescu A, Nutraceuticals. ( $1^{\text {st }}$ edn), Academic Press, 427-479.

20. Theodoratou E, Kyle J, Cetnarskyj R, Farrington SM, Tenesa A, et al. (2007) Dietary flavonoids and the risk of colorectal cancer. Cancer Epidemiol Biomarkers Prev 16: 684-693. 
21. Rossi M, Negri E, Talamini R, Bosetti C, Parpinel M, et al. (2006) Flavonoids and colorectal cancer in Italy. Cancer Epidemiol Biomarkers Prev 15: 1555-1558.

22. Djuric Z, Severson RK, Kato I (2012) Association of dietary quercetin with reduced risk of proximal colon cancer. Nutr Cancer 64: 351-360.

23. Ward HA, Kuhnle GG, Mulligan AA, Lentjes MA, Luben RN, et al. (2010) Breast, colorectal, and prostate cancer risk in the European Prospective Investigation into Cancer and Nutrition-Norfolk in relation to phytoestrogen intake derived from an improved database. Am J Clin Nutr 91: 440-448.

24. Cotterchio M, Boucher BA, Manno M, Gallinger S, Okey A, et al. (2006) Dietary phytoestrogen intake is associated with reduced colorectal cancer risk. J Nutr 136: 3046-3053.

25. Shin A, Lee J, Lee J, Park MS, Park Jw, et al. (2015) Isoflavone and Soyfood Intake and Colorectal Cancer Risk: A Case-Control Study in Korea. PLoS One 10: e0143228.

26. Wang L, Lee IM, Zhang SM, Blumberg JB, Buring JE, et al. (2009) Dietary intake of selected flavonols, flavones, and flavonoid-rich foods and risk of cancer in middle-aged and older women. Am J Clin Nutr 89: 905-912.

27. Knekt $P$, Järvinen R, Seppänen R, Hellövaara M, Teppo L, et al. (1997) Dietary flavonoids and the risk of lung cancer and other malignant neoplasms. Am J Epidemiol 146: 223230.

28. Knekt $P$, Kumpulainen J, Järvinen R, Rissanen $\mathrm{H}$, Heliövaara M, et al. (2002) Flavonoid intake and risk of chronic diseases. Am J Clin Nutr 76: 560-568.

29. Mursu J, Nurmi T, Tuomainen TP, Salonen JT, Pukkala E, et al. (2008) Intake of flavonoids and risk of cancer in Finn- ish men: The Kuopio Ischaemic Heart Disease Risk Factor Study. Int J Cancer 123: 660-663.

30. Goldbohm RA, Hertog MG, Brants HA, van Poppel G, van den Brandt PA (1998) Intake of flavonoids and cancer risk: a prospective cohort study. In: Armado R, Andersson $\mathrm{H}$, Bardocz S, Serra F, Polyphenols in food. Office for Official Publications of the European Communities, 159-166.

31. Arts IC, Jacobs DR Jr, Gross M, Harnack LJ, Folsom AR (2002) Dietary catechins and cancer incidence among postmenopausal women: the lowa Women's Health Study (United States). Cancer Causes Control 13: 373-382.

32. Oba S, Nagata C, Shimizu N, Shimizu H, Kametani M, et al. (2007) Soy product consumption and the risk of colon cancer: a prospective study in Takayama, Japan. Nutr Cancer 57: 151-157.

33. Yuan JM, Gao YT, Yang CS, Yu MC (2007) Urinary biomarkers of tea polyphenols and risk of colorectal cancer in the Shanghai Cohort Study. Int J Cancer 120: 1344-1350.

34. Ward H, Chapelais G, Kuhnle GG, Luben R, Khaw KT, et al. (2008) Lack of prospective associations between plasma and urinary phytoestrogens and risk of prostate or colorectal cancer in the European Prospective into Cancer-Norfolk study. Cancer Epidemiol Biomarkers Prev 17: 2891-2894.

35. Bobe G, Murphy G, Albert PS, Sansbury LB, Lanza E, et al. (2010) Serum cytokine concentrations, flavonol intake and colorectal adenoma recurrence in the Polyp Prevention Trial. Br J Cancer 103: 1453-1461.

36. Bobe G, Albert PS, Sansbury LB, Lanza E, Schatzkin A, et al. (2010) Interleukin-6 as a potential indicator for prevention of high-risk adenoma recurrence by dietary flavonols in the polyp prevention trial. Cancer Prev Res (Phila) 3: 764-775. 